\title{
Small Particles in Gas Suspension
}

\author{
New Experiments with Implications \\ in Basic and Applied Research
}

\author{
A. Schmidt-Ott and H. Burtscher, Zurich
}

(Laboratorium für Festkörperphysik, ETH)

Small particles, 2 to $10^{2} \mathrm{~nm}$ in diameter have attracted more and more interest in basic research during the last years. Being larger than atomic clusters and containing more than $10^{2}$ atoms, such particles show bulk behaviour in many respects: for example, they have a band-like electronic structure and a work function equal to that of extended material. However, large deviations in dielectric properties have been observed. Examples are Raman scattering from adsorbates on rough surfaces or small particles (surface enhanced Raman scattering), the photoelectric effect, van der Waals interaction and infrared absorption, all effects showing enhancements of several orders of magnitude compared to the flat extended surfaces.

Large differences with respect to bulk properties have also been derived theoretically. For instance, the polarizability $\alpha(\omega)$, determined by a self-consistent, quantum-mechanical jellium model calculation by W. Eckardt shows large deviations from the classical Mie theory. However, theories quantitatively matching experimental observations have been forthcoming in a few cases only. One reason for these discrepancies is the complexity or lack of definition of experimental conditions. It is common practice to make measurements on particles embedded in a substrate or matrix but the influence of these can scarcely be assessed.

To overcome this and avoid unnecessary contact with other material we have adopted the technique of suspending the particles in a gas. While particle production in the gas phase ("gas evaporation technique") has been practised for a long time in connection with their subsequent deposition on a substrate, experiments on the particles in the gas itself are relatively new (Schmidt-Ott and Siegmann, 1978). They were restricted to photoemission and coagulation until the first measurements of light scattering and fluorescence from a jet of $\mathrm{Ag}$ particles in $\mathrm{Ar}$ were performed just recently by Frank et al.

In contrast to gas suspension, particles concentrated in or on a solid or liquid lend themselves to many experimental techniques of solid state and sur- face physics, but the disadvantage of interaction already referred to cannot be avoided. Mention should also be made of investigations on particle beams in vacuum where particle sizes range from single atoms to several hundred $\AA$ in the latest experiments. Multiphoton ionization and electron impact have been applied, and the detection method is mass spectroscopy of the particles charged.

In order to relate gas suspension to other approaches, the following features of gas suspension are also worth noting: Adsorbates can be added at will, the temperature is defined and variable, size classification and the production of monodisperse aerosols is possible, and the particles can be held available for manipulations and observations for a long time, so long-term changes can be seen.

Moreover, the methods developed for the investigations of gas suspended particles have interesting applications in the analyses of airborne particles, i.e. in aerosol research. In particular, photoemission from gas-suspended particles can be of value in the analysis of ambient aerosols, especially man-made pollutants. In turn, aerosol research is providing theories and techniques that can now be applied to basic investigations. Among these are diffusion charging, size classification methods based on diffusion or mobility measurements and various particle production and detection principles.

While the importance of small atmospheric particles for cloud formation, precipitation, air conductivity, atmospheric chemistry and health has been recognized for a long time, methods for the microstructure analysis of aerosol particles 2 to $10^{2} \mathrm{~nm}$ in diameter are almost non-existent. Measurements frequently practised today yield the particle size distribution, the total particulate mass and a chemical analysis of collected particles. Information on the chemical composition of the surface is lost in the collection step although its importance in chemical, biological and physical processes is evident.

Surface-specific information has been obtained by complicated procedures like Auger electron spectroscopy from preci- pitated particles or mass spectrometry of molecules evaporated from single particles larger than $1000 \mathrm{~nm}$ heated by a laser beam. In both methods the changes occurring on the particle surface due to vacuum conditions and contact with the substrate or with each other can hardly be estimated. Near-UV photoemission from airborne particles may serve as an in-situ method in particle surface analysis and thus fills a gap in aerosol measurement.

Photoemission from Particles Suspended in a Gas

The three-step-model of Spicer describes photoemission from a large surface in vacuum, but for small particles in a gas the model must be modified and a fourth step added. The steps are:

1) Excitation of an electron by absorption of light: Absorption depends on the optical properties of the particle which are determined by its composition and geometry. Various size-effects leading to an enhanced absorption by particles have been reported.

2) Escape of an excited electron from the surface barrier potential: The electron's kinetic energy perpendicular to the surface must be larger than the photothreshold $\phi$. Near threshold, the yield $Y(h v)$ of emitted photoelectrons per incident photon is given by the Fowler-Nordheim equation:

$$
Y=c(h v-\phi)^{m}
$$

where $h v$ is the photon energy, and $m=$ 2 for metals. The constant $c$ depends on the electron excitation probability (step 1) and its escape probability. The latter can be enhanced up to a factor of four for particles small compared to the attenuation length of the electron, because escape is possible in all directions. While still larger enhancements of $c$ have been measured for small particles, the Fowler-Nordheim equation has been found to retain its validity.

3) Escape of an electron from the combined image and Coulomb potentials: The Coulomb attraction between the emitted electron and a particle carrying the remaining positive charge increases the photothreshold. Combining this with the image potential modified for a sphere as against a plane, the photo- 
threshold of a spherical particle of radius $R$, initially having $p$ elementary charges, is:

$$
\phi=\phi_{0}+\frac{e^{2}(p+1)}{4 \pi \varepsilon_{0} R}-\frac{5}{8} \frac{e^{2}}{4 \pi \varepsilon_{0} R}
$$

4) Escape of a photoelectron from the neighbourhood of the particle by diffusion: Whereas in photoemission from plane surfaces in gas at atmospheric pressure the probability of diffusion of the emitted electrons back to the surface is almost unity if no electric field is applied (since reabsorption of the electron by the surface can be assumed at the first collision), in photoemission from a small particle, the situation is quite different. In practice, the escape probability is close to unity if the particle is small compared to the electron mean free path in the gas, and this can be arranged by the choice of gas pressure. To be precise, the probability of electron liberation depends also on the gas molecule cross-sections for elastic and inelastic scattering and capture of electrons (negative ion formation), as well as the molecular mass, but for particles smaller than $100 \mathrm{~nm}$ in diameter, for example, the escape probability can be assumed unity at $1 \mathrm{~atm}$.

Another mechanism to be considered is the attachment of emitted electrons or negative ions to other particles. The corresponding probability can be determined by a theory due to Hopple but again may be made negligible if the concentrations of particles and negative carriers are kept small.

Measurement Principles of Photoemission from Gas Suspended Particles

\section{Aerosol Photoconductivity}

The microscopic charge separation caused by emission of electrons from particles can be determined by measurement of the corresponding conductivity. A set-up designed for this purpose is shown in Fig. 1.

In order to avoid field-induced macroscopic charge separation and loss of charge carriers by neutralization at the electrodes, the field is alternating and the differential in-phase current component of illuminated and dark aerosol is measured. In this way, the additional gas conductivity arising from radioactivity and cosmic rays is eliminated. A sensitivity of 50 electrons per $\mathrm{cm}^{3}$ has been achieved.

Although the method is, in principle, applicable to clean inert gas, it has, so far, only been applied to gases containing an electronegative component like $\mathrm{O}_{2}$ which captures the photoelectrons which thereby assume a defined mobility. A quantitative evaluation is then pos-

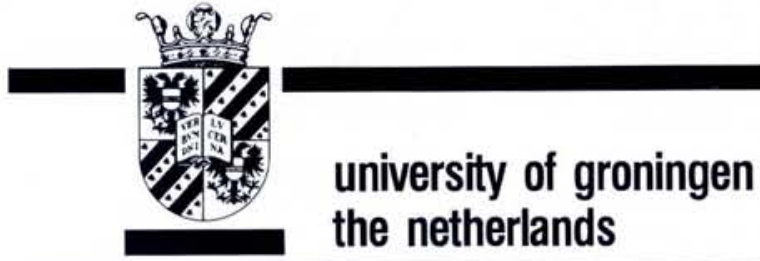

\section{- temporary position in the theory of condensed matter}

at the Department of Physics.

Within the process of formation of a new Group in the Theory of Condensed Matter and Statistical Mechanics a temporary position is available in the Solid State Physics Laboratory. The present research activity of the group concerns mainly fractal structures and charge density waves as well as other projects linked to the research programs of the Materia Science Centre of the Faculty of Science. The position is research oriented with a minor didactical activity.

The appointment is for two years with the possibility of extention up to four years. Salary depending on age and experience to a maximum of Dfl. 5.534 . per month.

Further information can be obtained from prof.dr. L. Pietronero, Solid State Physics Laboratory, tel. 050-115422.

Applications, including a curriculum vitae, a list of publications and the names of two referees, should be sent to the Head of the Personnel

Department, University of Groningen, P.O. Box 72, 9700 AB Groningen,

The Netherlands, within three weeks after publication of this announcement, quoting our reference number $841012 / 2493$. sible. Furthermore, the loss rate of "photoelectrons" by diffusion to the electrodes is lowered substantially, allowing a time - resolved conductivity measurement to be made. After removal of the irradiating source, the conductivity decays according to the diffusion losses of the charge carriers. This enables the diffusion constants of ions and particles, and hence the particle size to be determined. The conductivity yields the absolute photoelectric quantum yield $Y(h v)$. In the presence of $\mathrm{O}_{2}$, the photon energy is restricted to $h v<6 \mathrm{eV}$, since substantial absorption by the gas occurs above this energy.

\section{Gas Multiplication}

An extremely sensitive technique of photoelectron detection is based on Townsend avalanche gas multiplication; single photoelectrons from $10 \mathrm{~nm} \mathrm{Ag}$ particles have been detected by this method. In the inhomogeneous field near the surface of a thin anode sense wire, a

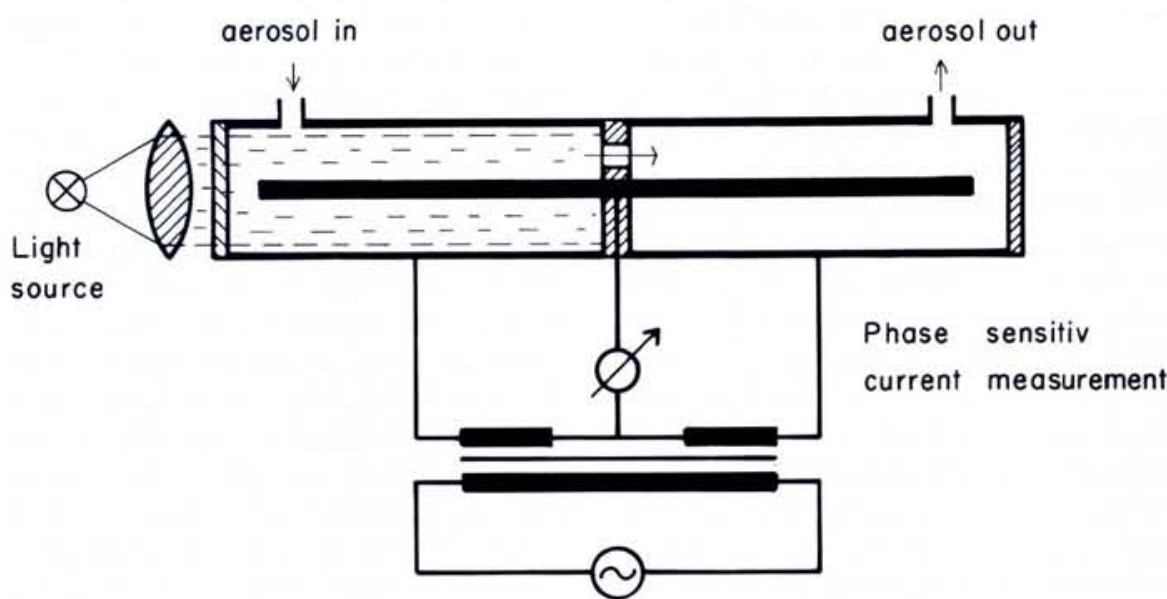

Fig. $1-$ Cylindrical chamber with circuit for aerosol photoconductivity measurement.

Townsend avalanche is produced from a single electron and the resulting current pulse is detected. A disadvantage of the method is the need to have present a quench gas to avoid a break-down discharge. While the quench gas may be chosen such that the incident radiation is not attenuated, it will, in general be adsorbed at the particle surface as an unfavourable contaminant. For this reason, a technique applying short high-voltage pulses not leading to break-down is presently being tested.

\section{Macroscopic Charge Separation}

Another measuring principle for photoemission from gas suspended particles is based on the separation of photoelectrons creating negative ions from the particles and measuring the current of either in a continuous aerosol flow. For the separation one can take advantage of different diffusion mechanisms. The negative carriers diffuse to the wall where they are neutralized and the par-

aerosol out 
ticles follow the flow and can be collected in a fibre filter mounted in a Faraday cup. Separating the charges in an electric field is another possibility.

An apparatus designed for basic studies on metal particles with defined surfaces under inert gas conditions is shown in Fig. 2. Helium is used as a carrier gas since it can be obtained extremely clean, by evaporating from the surface of liquid $\mathrm{He}$, where all but $\mathrm{He}$ condenses. Furthermore, He shows no light absorption in the spectral range of interest. In order to ensure maximum purity the whole system is baked and evacuated to a pressure of $<10^{-9} \mathrm{mbar}$ before flooding with $\mathrm{He}$. A continuous flow of $\mathrm{He}$ heated to room temperature $(\sim 4$ $\ell / \mathrm{min}$ ) is then maintained at a pressure of $1 \mathrm{bar}$ and carries the particles produced in the aerosol source through the system. Particles are formed by evaporation of the metal to be investigated and homogeneous nucleation. An electrostatic prefilter removes all charged particles. In the following step the photoemission chamber is irradiated with monochromatic light of a photon energy of $4-10 \mathrm{eV}$. The photoemitted electrons diffuse to the walls or attach themselves to other particles. The radiation intensity is kept so low that only a small fraction of particles emit electrons, avoiding recombination and double charging. In the following electrostatic filter the positive particles are precipitated onto an electrode and the corresponding current is measured. It is directly proportional to the photoelectric quantum yield $Y(h v)$. Since the precipitation rate depends on the particle mobility, the particle size can be estimated from the current-voltage characteristic. The experiment provides only a relative determination of $Y(h v)$ since the particle concentration is not known.

\section{Results of Basic Interest}

In the following, some results obtained by the experimental techniques previously described are summarized.

1. The Fowler-Nordheim law (eq. (1)) has been confirmed near threshold for particles of $\mathrm{Ag}, \mathrm{Au}, \mathrm{MoO}_{3}$, and $\mathrm{WO}_{3}$ down to diameters of $40 \AA$.

2. Table 1 shows the photothreshold $\phi$ and the yield constant $c$ (eq. (1)) of $\mathrm{Ag}$ particles of mean radius $R$ produced by evaporation from a Ag wire in $\mathrm{N}_{2}$. They were obtained in an aerosol photoconductivity measurement in a $\mathrm{N}_{2}-\mathrm{O}_{2}$-mixture and compared with the values measured by photoemission from the surface of this wire in the same gas mixture without particles. The strong increase of $c$ with decreasing particle size and an enhancement of up to $10^{2} \mathrm{com}$ -
Fig. 2 - Apparatus for measurement of photoemission from small particles under clean conditions.

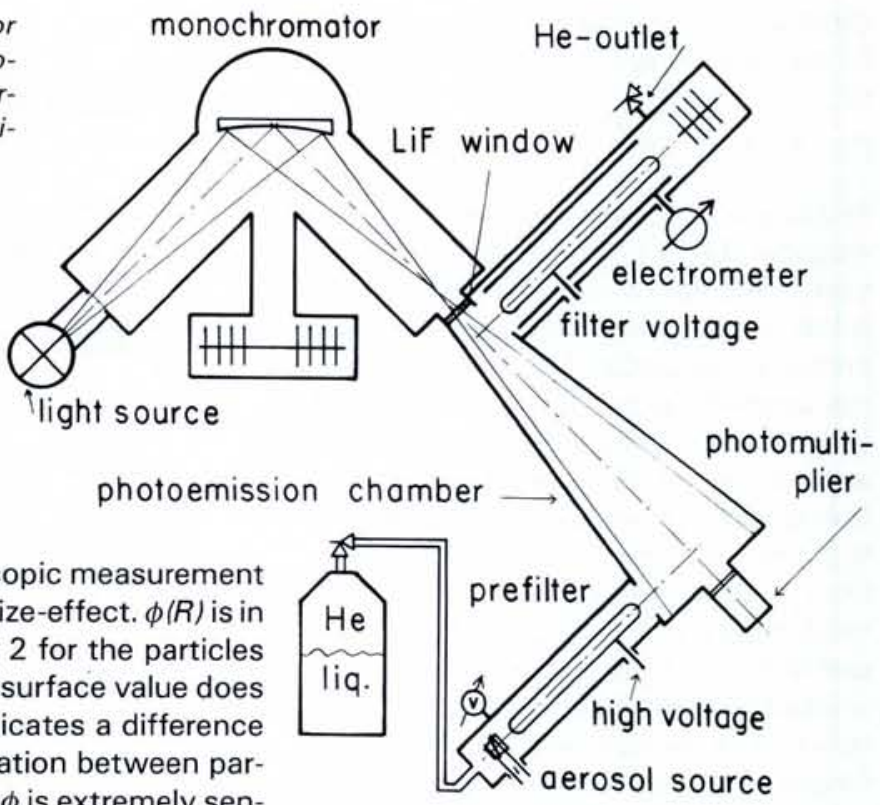

ticles and wire, since $\phi$ is extremely sensitive to the surface dipole layer. Moreover, beam experiments as well as our own findings under clean conditions (q.v.) have clearly shown that $\phi$ is not size-dependent for the sizes we deal with.

3. In Fig. 3 is given a comparison of $Y(h v)$ of clean $\mathrm{Ag}$ particles of radii $R=$ $50 \pm 20 \AA$ in $\mathrm{He}$ with $Y(h v)$ for bulk $\mathrm{Ag}$ under ultra-high vacuum conditions. For easy comparison, bulk and particle yields have been normalized to overlap at the high-energy end of the curve. While the bulk curve has no marked structure the particle curve shows a steeper rise above threshold followed by an almost flat section between 7 and $8.5 \mathrm{eV}$. Another enhanced slope occurs where emission from the d-bands starts. Here the difference in the photothresholds between particles and bulk (not displayed in the graph) are in accordance with eq. 2 , confirming clean particle surfaces. The same measurement for $A u$ particles shows no significant difference between the bulk and particle yields.

The effect of traces of undefined contamination of the particle surface is studied by using $\mathrm{He}$ from an ordinary high pressure gas bottle partially cleaned in a liquid $\mathrm{N}_{2}$ cooling spiral. A dramatic change of $\phi$ is seen, indicating a potential step at the surface due to adsorbates. Surprisingly, $Y(h v)$ increase up to one order of magnitude under contamination. While a $Y$-decrease induced by contamination is a well-known phenomenon and can be ascribed to electron scattering from the surface adsorbate, an increase of $Y$ by contamination may represent a size effect. It points to the crucial role of the surface electron density profile which is in qualitative agreement with theoretical findings. Moreover, the large sensitivity of $Y$ to contamination suggests that much of the enhancement referred to previously may also have been brought about by the difference between adsorbates on particles and macroscopic surfaces.

Measurements involving additions of trace amounts of defined adsorbates are in preparation. Such measurements should shed more light on the role of the surface in the observed size effects. Furthermore, the degree of adsorption can principally be monitored by measurement of $\phi$ and $Y(h v)$ and reactions taking place at the particle surface will further modify these quantities in a characteristic way, allowing their observation. This should be of great interest for the study of heterogeneous catalysis by small particles.

\section{Van der Waals Forces and Aerosol Coa- gulation}

Another size effect found in gas suspended particles is worth mentioning. Van der Waals, or dispersion forces are closely related to dielectric properties in the optical range. In addition to the photoyield enhancements, large infrared absorption enhancements of small particles have been reported by Devaty and Sievers. Where abnormal optical behaviour occurs, abnormal van der Waals forces must be expected.

Table 1 - Ag Photothreshold and Yield Constant against Radius in $\mathrm{N}_{2}$

\begin{tabular}{|l|c|c|c|}
\hline & $R, \AA$ & $\emptyset, \mathrm{eV}$ & $c$ \\
\hline Particle & 30 & 4.55 & $1.8 \times 10^{-3}$ \\
& 27 & 4.57 & $3.9 \times 10^{-3}$ \\
& 20 & 4.65 & $8.9 \times 10^{-3}$ \\
\hline Macroscopic & & & \\
surface & $\infty$ & 4.90 & $7.3 \times 10^{-5}$ \\
\hline
\end{tabular}


A measurable phenomenon related to these forces between particles is coagulation, which is detectable by the measurement of particle size or concentration. Collision probability is increased by forces between the particles, so that the measurement of the time dependence of size and concentration of neutral particles will lead to a lower limit for the van der Waals forces on making the assumption that every collision results in bonding.

Size and/or concentration have been determined by photoconductivity or mobility analysis. The measurements have led to the result that van der Waals forces between $14 \mathrm{~nm}$ Ag particles in an $\mathrm{O}_{2}-\mathrm{N}_{2}$-mixture are enhanced by at least a factor of $10^{4}$, whereas $\mathrm{C}$ particles show normal behaviour.

\section{Particulate Airborne Pollutants}

In the analysis of atmospheric aerosols, particles originating from the combustion of organic material like oil, gasoline, wood and garbage are of special interest. Numerous experiments have shown that such particles exhibit an unusually high photoelectric activity $\varepsilon$ (photoemission probability per particle per second). Further investigations have shown that the highly photoemissive species are hydrocarbons exposed on the particle surface. This is consistent with the results of chemical analysis of such particles which indicates that they contain a large variety of aliphatic and aromatic hydrocarbons. Experiments with artificial hydrocarbon coated aerosol particles have shown that small amounts of polyaromatic hydrocarbons condensed on the surface of $\mathrm{NaCl}$ or $\mathrm{C}$ particles lead to the highest $\varepsilon$ ever

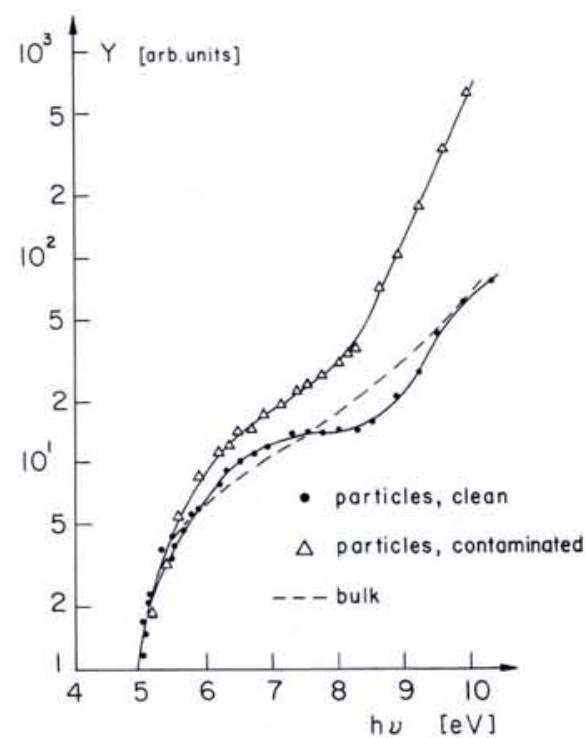

Fig. 3 - Photoelectric yield of clean and contaminated Ag particles and bulk Ag vs. photon energy.

\section{the Delft University of technology, the Netherlands}

In the Department of Applied Physics, Solid State Group, a position is available for a

\section{professor of experimental semiconductor physics}

Candidates must be experienced in experimental research on solid-state physics of semiconductors. It is expected that new research is started which preferably has connections with other activities in the department. Examples of possible subjects are: optical phenomena in semiconductors or the physics of semiconductor structures with submicron dimensions. Other subjects are certainly not excluded. The presence of the Centre for Submicron technology in the same building provides interesting possibilities.

Candidates are invited to apply, within a month after the date of publication to the chairman of the selection committee, prof. J.J.J. Kokkedee, Department of Applied Physics, Lorentzweg 1, $2628 \mathrm{CJ}$ Delft, The Netherlands.

Information can be obtained with the chairman, telephone 31-15-784117

or prof. J.E. Mooij, telephone 31-15-786153.

observed for aerosol particles. Moreover, garage aerosol samples exhibit an $\varepsilon$ of the same order of magnitude. The subject is of particular interest because of the high biological activity of some polyaromatic hydrocarbons. Among them, benzo(a)pyrene is considered as the most mutagenic and carcenogenic component.

Particles originating from combustion are found in garage or city air probes. The size dependence of their photoelectric activity has been measured by combining one of the techniques previously described with a size classification by mobility analysis. For most probes, $\varepsilon$ was fairly well proportional to the particle diameter $D_{\mathrm{p}}$. This is consistent with a model of particle formation in which the photoelectrically active component is the most volatile, condensing last on the particle surface and thus being present only at the surface. According to diffusion theory, the amount of this component deposited is proportional to the particle radius. Yet combustion under very $\mathrm{O}_{2}$ - deficient conditions, leading to a relatively large amount of hydrocarbons, produces particles from which the emission is proportional to the surface $\left(\varepsilon \sim D_{p}{ }^{2}\right)$. Here one must presume that so much photoelectrically active material has condensed on the particle that the surface is totally covered with it. An important implication of these observations is that in general, atmospheric particulate pollutants are only partially covered by the active material which indicates that the total amount present may be small compared to the total aerosol mass. It is the bulk characteristics that are analyzed in conventional techniques, whereas most of the important properties of the airborne particles are determined by these traces of surface material.

Such studies underline the necessity for a surface sensitive technique analyzing the particles in their natural environment. Aerosol photoemission measurements can be extended to allow chemical classification. Pilot studies including the addition of gaseous reactants and particle surface evaporation by variation of aerosol temperature in combination with photoemission have already shown that certain hydrocarbons can be distinguished.

\section{BIBLIOGRAPHY}

Photoemission from Small Particles:

Burtscher H., Scherrer L., Siegmann H.C., Schmidt-Ott A. and Federer B., J. Appl. Phys. 53 (1982) 5.

Schmidt-Ott A. and Federer B., Surf. Sci. 106 (1981) 538.

Schmidt-Ott A. and Siegmann H.C., Appl. Phys. Lett. 32 (1978) 710.

Seiler P.G., Burtscher H. and Schmidt-Ott A., Nucl. Instr. Meth. 203 (1982) 129.

\section{Size Effects:}

Bennemann K.H. and Reindl S., Berichte der Bunsengesellschaft, Vol. 88/3 (1984).

Burtscher $\mathrm{H}$. and Schmidt-Ott A., Phys. Rev. Lett. 48 (1982) 25.

Devaty R.P. and Sievers A.J., Phys. Rev. Lett. 52 (1984) 1344.

Eckardt W., Phys. Rev. Lett. 52 (1984) 21.

Frank F., Schulze W., Tesche B., Urban J. and Winter B., to be published in Surf. Sci. (1984).

Schmidt-Ott A. and Burtscher H., J. Colloid Interface Sci. 89 (1982) 353.

Schmidt-Ott A., Schurtenberger P. and Siegmann H.C., Phys. Rev. Lett. 45 (1980) 15.

\section{Coated Particles:}

Burtscher H. and Schmidt-Ott A., Sci. Tot. Env. 36 (1984) 233. 